\title{
Safe Testing of Electrical Diathermy Cutting using a New Generation Soft Manipulator
}

\author{
Francisco J. Comin ${ }^{1}$ Member, IEEE, Chakravarthini M. Saaj ${ }^{1 \dagger}$ Senior Member, IEEE, \\ Seri M. Mustaza ${ }^{1}$ and Rajendran Saaj ${ }^{2}$
}

\begin{abstract}
First demonstration of a pneumatic soft continuum robot integrated in series with a rigid robot arm safely performing tele-operated diathermic tissue cutting. The rigid arm autonomously maintains a safe tool contact force, while the soft arm manually follows the desired cutting path. Ex-vivo experimentation demonstrates sub-millimetric deviations from target paths.
\end{abstract}

Index Terms - Medical Robots, Minimally Invasive Surgery, Flexible Arms, Force Control, Diathermy Cutting.

\section{INTRODUCTION}

$\mathrm{R}$ OBOTIC solutions can add significant value to the field of Minimally Invasive Surgery (MIS) procedures [1], [2]. MIS reduces the trauma and recovery time for patients, while robot-assisted surgery has the potential to greatly extend MIS techniques to hitherto restricted sites and conditions. Traditional surgical robots are rigid laparoscopic tools, with a few articulated degrees of freedom [3], which suffer from a limited maneuverability range and risks of tissue damage.

Continuum robots overcome the first limitation by drastically incrementing the number of degrees of freedom, combining many micro-actuated links in series [4] to achieve hyperredundant motion. Alternatives like cable-driven catheters [5], [6] or pneumatic manipulators [7], [8] can partially reduce the inherent complexity of such a high number of actuators and improve safety through the compliance of their soft bodies. However, this implies significant material deformations [9] and viscoelastic effects [5] that make modeling the kinematics [10][13] and dynamics [14]-[16] of soft continuum robots challenging. Said complexity affects real-time control of such a surgical robot, which constitutes one of the main open research fronts in this field together with reliable direct contact force sensing [6] and feedback for palpation [17] and grip control [18], imaging and localization of the surgical robot within the patient [19]-[21], and tele-operation of the robot [22]-[24].

The rigid nature of conventional surgical robotic arms makes manipulation in tortuous body cavities and surgery around important organs difficult. This means the surgeon might not be

Paper submitted for review on 27/05/2017. This work was supported in part by the STIFF-FLOP* project grant from the EU Seventh Framework Program under grant agreement 287728 and by University of Surrey funds.

${ }^{*}$ STIFFness controllable Flexible and Learn-able manipulator for surgical OPerations. http://www.stiff-flop.eu/

${ }^{\dagger}$ Corresponding author. able to apply MIS, being forced to resort to more traumatic open surgery techniques. Flexible robotic arms have a larger potential to overcome this limitation, making MIS an option for more cases, e.g. complex surgery in the throat, chest or abdominal cavity and gynaecological operations. This paper demonstrates successful integration of a rigid-link arm for tool insertion with a soft manipulator for safe tissue interaction, focusing on the application of diathermy to controlled tissue cutting. A pneumatic soft continuum robot is used, emphasizing the quantitative analysis of cutting performance, as opposed to previous work on similar tasks which made use of catheter-like robots [25], rigid hyper-redundant robots [4] and simulations [24] or made no assessment of cut accuracy [6], [26]. The goal is to prove that the higher potential for tortuous maneuverability of flexible robots does not involve a loss of the end-effector teleoperation accuracy demonstrated by rigid-link arms.

The soft continuum manipulator considered in this research was developed as part of the EU FP7 STIFF-FLOP Project [27], [28]. It is conceived as a modular device [27], [29], inspired by octopus' tentacles [30]-[32]. It is composed of serially connected modules fabricated from Ecoflex 0050 silicone, pictured in Fig. 1(a), and are individually actuated by regulating the pressure of three internal chambers shown in the crosssection of Fig. 1(c). The undesirable ballooning effect observed at higher pressures was initially restrained through an external sleeve [33], and was later substituted by internal braiding of the individual chambers to eliminate existing sensing and actuation issues [34]. In addition, the modules can be provided with a stiffening mechanism based on vacuum-actuated granular jamming [35], [36] of additional internal chambers filled with ground coffee granules, shown in Fig. 1(d). The design and fabrication processes of the modules are not a contribution of this paper and can be found with more detail in [28]-[34].

The rest of the paper is organized as follows: Section II presents the high level task decomposition for the integrated system. Section III describes the models used to control the system. Section IV introduces the ex-vivo experimental set-up and methodology. Section $\mathrm{V}$ analyzes and discusses the ex-vivo experimental results. Section VI summarizes the main conclusions and future work directions.

\footnotetext{
${ }^{1}$ Francisco Comin, Chakravarthini Saaj and Seri Mastura Mustaza are with the Department of Electronic Engineering, Faculty of Engineering and Physical Sciences, University of Surrey, Guildford, Surrey, GU2 7XH UK (e-mail: \{ f.comin;c.saaj;s.mustaza\}@surrey.ac.uk).

${ }^{2}$ Rajendran Saaj was with the Royal Free London Hospitals NHS Trust, UK. (e-mail: dr_saaj@yahoo.co.uk).
} 


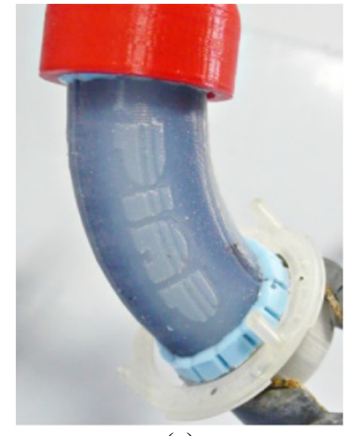

(a)

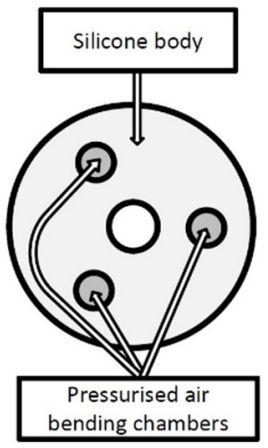

(c)

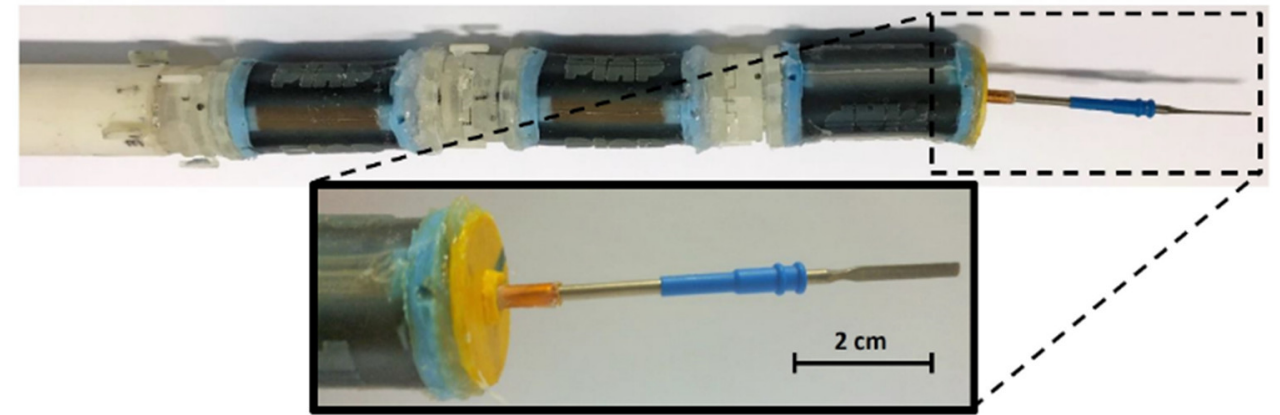

(b)

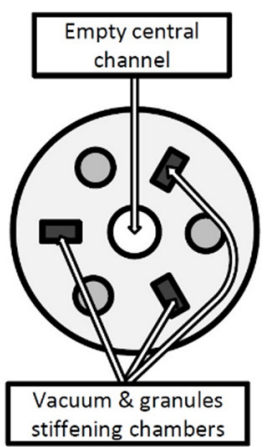

(d)

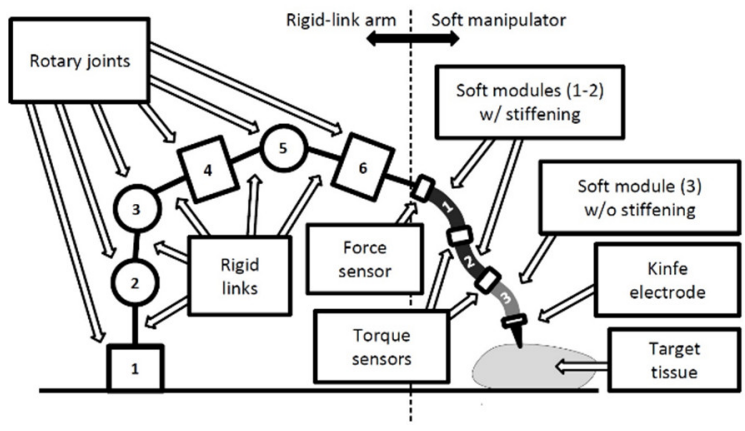

(e)

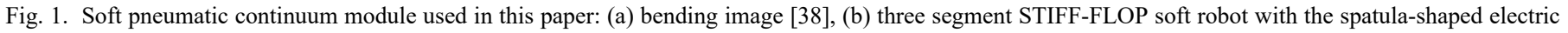

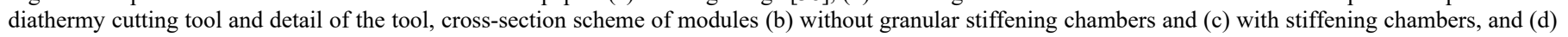
diagram of integrated soft robotic system with rigid-link arm and knife electrode tool (not to scale).

\section{High LeVEL TASK DeCOMPOSITION}

The system proposed is formed by two main, highly heterogeneous robotic sub-systems: 1) a rigid-link arm with six degrees of freedom and a sufficiently large workspace in order to gain access to any potential entry point to the patient's body on the operating table, and 2) a soft continuum manipulator with enough flexibility to safely maneuver within the patient's body and reach the surgery location, while providing the stiffness needed to carry the surgical instrument and maintain its position (see Fig. 1(a) to 1(d)).

The integrated overall system and its different components are represented in Fig. 1(b). Each of these sub-systems employs different actuation strategies and sensors depending on the different stages of the electrical diathermy cutting procedure addressed in this paper. The soft robot shown in Fig. 1(b) is made up of three identical modules each $5 \mathrm{~cm}$ long. The overall dimension of the soft robot including the three inter-module connectors but excluding the cutting tool is about $20 \mathrm{~cm}$ in length under rest position.

\section{A. Positioning of Rigid-Link Arm}

The first task phase consists of moving the rigid-link arm outside the patient's body, from its initial configuration to the desired insertion pose through a laparoscopy port. The goal and trajectory are pre-programmed when planning the surgery, taking into account the location of the body, entry point and the length of the soft manipulator attached to its end-link. The planned path is followed by the rigid arm based on the speed and position feedback from its sensors, using the standard Inverse Kinematics (IK) model of the robot arm to calculate the joint speed commands $(\dot{\vartheta})$ necessary to follow the desired trajectory in differential Cartesian coordinates of the fixed workspace reference frame $\left(\dot{q}_{W}\right)$ through the inverse of the corresponding Jacobian matrix $\left(J_{W}\right)$ as in Eq. (1).

$$
\dot{\vartheta}=J_{W}^{-1} \dot{q}_{W}
$$

During this stage, the soft manipulator only reacts passively to the effect of gravity, maintaining its tip aligned with the forward direction of the arm's end-link so as to facilitate insertion once the entry location is reached. This can be achieved by measuring inter-module forces and torques [37], with the stiffness tuning method proposed in [38].

\section{B. Insertion, Approach and Contact}

The second phase encompasses the insertion of the soft manipulator within the body of the patient. The motion of the rigid arm is constrained to linear translation along and roll rotation around the axis of insertion. Therefore, bending the soft manipulator might be necessary in order to face the operation location and/or avoid any en route organs/structures until contact is made with the targeted tissue.

During this stage, the forward motion of the rigid arm is manually enabled by the operator and is automatically driven by the contact force, so as to ensure a safely bounded contact force. Backward motion is manually driven by the operator whenever retraction is necessary.

The desired bending of the soft manipulator can be achieved through manual tele-operation combining visual position feedback and a 3D Inverse Kinematics (IK) model of the soft manipulator, as proposed in [13]. 


\section{Soft Manipulator Controlled Cutting}

Once the desired contact force and relative location between the end of the soft manipulator and the targeted tissue are achieved, the third and final phase of the task is entered. The stiffness of the current bent configuration of the soft manipulator is increased through the activation of the granular jamming in the first and second modules.

Meanwhile, the second and third modules are actuated in the desired cutting direction, activating the electro-knife to perform the cut. The normal contact force is maintained through the linear insertion/extraction motion of the rigid arm, automatically compensating any normal elongation/shortening of the bent soft manipulator and reducing the effective dimensionality of the cutting motion to the surface of the tissue.

It is important to note that the activation of the stiffening elements generally does not produce a significant change in the shape of the first two modules, but rather helps them maintain the desired shape at the moment of activation. However, on occasions, a slight change of shape can be momentarily observed upon activation. Nonetheless, this activation is performed only once tool-tissue contact is already established and contact force control is active. Therefore, these slight shape changes did not result in deviations of the tool-tissue contact position or unsafe contact forces.

\section{MODELLING AND CONTROL}

The following models are used to control the motion of the system through the different task stages.

\section{A. Contact Force Regulation}

During the second and third phase of the task, the position of the rigid-link arm is no longer controlled relative to the workspace reference frame, but rather to its local end-effector reference frame $\left(\dot{q}_{E}\right)$. In addition, external constraints are added, so that the rigid arm is only allowed to move along and rotate around that frame's Z-axis $\left(\dot{x}_{E}=\dot{y}_{E}=\dot{\delta}_{E}=\dot{\psi}_{W}=0\right)$.

As a result, the IK problem is now solved through the endeffector frame Jacobian matrix $\left(J_{E}\right)$ using only the insertion/retraction $\left(\dot{z}_{E}\right)$ and roll $\left(\dot{\varphi}_{E}\right)$ speeds as inputs. Since the system is now redundant, the pseudoinverse of the Jacobian matrix can be used following Eq. (2).

$$
\dot{\vartheta}=J_{E}^{T}\left(J_{E} J_{E}^{T}\right)^{-1} \dot{q}_{E}=J_{E}^{+}\left[\begin{array}{ll}
\dot{z}_{E} & \dot{\varphi}_{E}
\end{array}\right]^{T}
$$

The roll angular speed is directly controlled by the operator, in order to re-orient the soft manipulator during insertion/cutting in phases two and three. On the other hand, the insertion/retraction linear speed is automatically generated by a Proportional, Integral and Derivative (PID) impedance controller, which takes the indirectly measured contact force $(F)$ as an input and drives it towards a desired force reference value $\left(F_{R}\right)$ by inserting/retracting the end-effector of the rigid arm to compensate insufficient/excessive forces, applying Eq. (3). The gains of the controller $\left(k_{P}, k_{I}\right.$ and $\left.k_{D}\right)$ are tuned to achieve settling times below 2 seconds and to maintain forces below a $1 \mathrm{~N}$ maximum safety threshold.

$$
\dot{z}_{E}=k_{P}\left(F_{R}-F\right)+k_{I} \int\left(F_{R}-F\right) d t+k_{D} \frac{d\left(F_{R}-F\right)}{d t}
$$

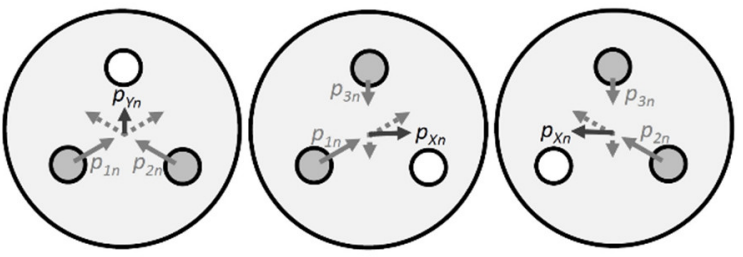

Fig. 2. Two-dimensional abstraction of the planar soft-module push as the geometrical superposition of individual chambers.

\section{B. Soft Manipulator 2D Cutting Motion}

During the third phase, once the cutting tool is in contact with the target tissue and tracking of the desired path is started, the contact is maintained and the associated force regulated automatically by the rigid-link, as described in Eq. (3). In consequence, the teleoperation of the soft manipulator can be focused solely on displacing its end-effector across the surface following the desired path. The components of the planar displacement generated by the $\mathrm{n}^{\text {th }}$ soft module $\left(p_{X n}\right.$ and $\left.p_{Y n}\right)$ through the bending/elongation produced when actuating its chambers can be estimated as the geometric superposition of the individual internal chamber contributions $\left(p_{1 n} / p_{2 n} / p_{3 n}\right)$ through equation Eq. (4), using the two-dimensional abstraction depicted in Fig. 2.

$$
\begin{aligned}
& \dot{p}_{X n}=\dot{p}_{1 n} \cos \frac{\pi}{6}-\dot{p}_{2 n} \cos \frac{\pi}{6} \\
& \dot{p}_{Y n}=\dot{p}_{1 n} \sin \frac{\pi}{6}+\dot{p}_{2 n} \sin \frac{\pi}{6}-\dot{p}_{3 n} \\
& \dot{p}_{Z n}=\frac{\dot{p}_{1 n}+\dot{p}_{2 n}+\dot{p}_{3 n}}{3}
\end{aligned}
$$

The third component $\left(p_{Z n}\right)$ corresponds to the average internal pressure of all three chambers, and prevents the inverse system from being underdetermined, so that a unique solution for the internal chamber pressures can be calculated. Variations of this last component translate in changes of manipulator elongation rather than of the normal contact force, since the rigid arm force regulation passively accommodates for these length changes to preserve the desired contact force.

Throughout this phase, the activation of the granular jamming in the first two modules, as described in sub-section II.C, keeps their stiffness and elongation roughly constant, providing a rigid enough base for the bending of the third module to carry out the cutting action. The stiffness and elongation of the third module, while not explicitly included in the model, are indirectly accounted for by the third component of Eq. (4). Moreover, as explained in sub-section III.A, the automatic insertion/retraction of the rigid-link arm to regulate the contact force passively compensates any change in elongation of the soft manipulator, i.e. without requiring an explicit elongation estimate.

When the diathermy cutting tool is activated, the commanded displacement will cause the knife electrode to cut in the desired direction as the soft manipulator bends in said direction. If the tool has a preferential cutting direction, as is the case of the spatula-shaped electrode used in this paper, only the aligned push component ( $p_{X n}$ or $p_{Y n}$ depending on the configuration) will be effective in advancing the cut, while the cut direction will be controlled by the teleoperated roll rotation of the rigid-link arm. 


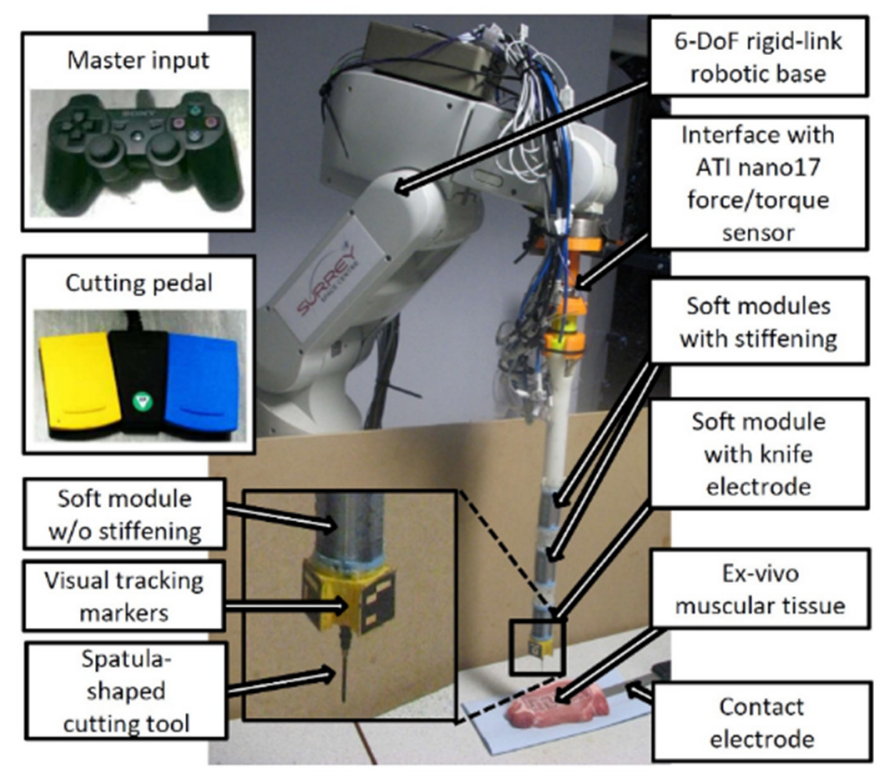

Fig. 3. Experimental setup with the integrated hardware system including the rigid arm and soft manipulator

\section{EXPERIMENTAL METHODOLOGY}

The described task and control paradigms are implemented in a physical platform for experimental validation. The aim is not only to demonstrate its functionality, but also to quantitatively assess its ability to follow ex-vivo cutting trajectories.

\section{A. Hardware Components and Interfaces}

A labeled image of the integrated experimental setup is shown in Fig. 3. The rigid-link arm used for experimentation is the MELFA RV-1a robot arm. It comes with six rotary joints in a yaw-pitch-pitch-roll-pitch-roll sequence, enabling full 6 degree-of-freedom pose control of its end-effector over a workspace volume of approximately $10^{5} \mathrm{~cm}^{3}$. The position of each joint is controlled by a proprietary Mitsubishi controller, which also provides real-time feedback of the angular position, speed and torque of each joint. The controller box is programmed using the MoveMaster language to relay position commands and joint feedback from/to a computer at a $70 \mathrm{~Hz}$ rate, via-Ethernet.

A 6-axis force/torque ATI Nano17 sensor, with a force resolution down to $1.46 \mathrm{mN}$, is rigidly attached to the endeffector of the rigid-link arm. The analogue outputs of this sensor are fed to a Shadow Robot Company RoNeX module, which samples the signals and transmits the measurements to the computer terminal at high speeds through an EtherCAT connection. The soft manipulator is rigidly attached to the other end of the ATI Nano17 sensor. The configuration used consists of three modules, each with three pneumatic chambers individually actuated by means of Camozzi K8P pressure micro-regulators, modulating a $150 \mathrm{kPa}$ air inlet according to Pulse Width Modulation (PWM) outputs produced by the RoNeX module [39]. The two first modules are provided with three additional granular jamming chambers like those illustrated in Fig. 1(d) and are activated through RoNeX-driven Camozzi vacuum regulators, as well as a RoNeX-sampled 2axis flexion torque sensor [37] linking both modules.

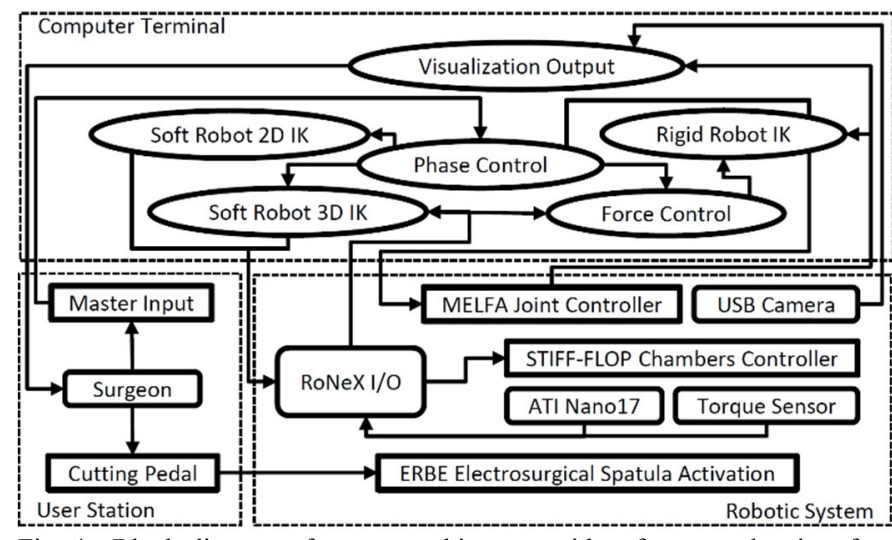

Fig. 4. Block diagram of system architecture with software nodes, interface hardware components and sensing/actuation elements.

The knife electrode of an ERBE VIO 50C electrosurgical system is fixed at the end of the third module, routing its connection through the central channel of the soft manipulator and the link chain of the MELFA arm. Three fiducial markers are placed at 120 degrees around the knife at the end of the third module, captured by a contextual USB camera for pose tracking using the ARUCO library [40]. In a more realistic scenario, e.g. in-vivo testing or an actual MIS procedure, this USB camera could be replaced by a laparoscopic camera. Such a real-time contextual visualization is crucial for the user to accounting for the slight delay of pneumatic actuation and soft module deformation relative to traditional rigid manipulator joints.

The user interface is provided by the master input device, manually controlled and provided with 10 configurable buttons, for high-level task control, and two joysticks, for tele-operation. The only exception is the activation of the electrosurgical tool, which is performed through the cutting pedal provided by ERBE. Both input devices are shown inset on Fig. 3.

\section{B. Software Architecture}

The software used to operate the system was implemented using the Robot Operating System (ROS) framework. The main nodes conforming the architecture, as depicted in the block diagram of Fig. 4, have the following functionalities:

Phase Control: Reads the inputs from the master input device and uses those inputs both for tele-operation and to control transitions between task phases and operation modes. According to the current task phase, control mode and the other inputs from the master input device, the node generates the corresponding actuation commands for the soft manipulator and the MELFA rigid arm.

Force Control: During phases 2 and 3, while not in manual retraction mode, this node generates the forward/backward motion command for the rigid arm according to the ATI Nano17 reading from RoNeX and the desired force.

MELFA IK: Solves the IK model for the MELFA rigid arm to produce the joint speed commands according to the feedback from sensors and the desired pre-programmed path during phase 1 , the output of the force regulator during phase 2 or the manual retraction/roll during phases $2 / 3$.

Soft Robot 3D IK: Generates the chamber pressure commands required to achieve the desired bending of the soft manipulator according to the flexion torque measurements 
during phase 1 as proposed in [38] or the inputs from the master input device during phase 2 as proposed in [13].

Soft Robot 2D IK: During phase 3, this node generates the chamber pressure levels necessary to yield the desired cutting action with the soft manipulator according the reduced twodimensional IK model presented in Section III.B.

Output Visualization: Receives the joint position feedback from the MELFA rigid arm and the visual feedback from the USB camera to generate a contextual video stream and a 3D visualization of the system's position in real time to guide the operator.

\section{Ex-Vivo Tissue Cutting using Electric Diathermy}

Electric diathermy is a widely used and versatile tool for surgeons of all specialties. It is used to safely cut tissue and organs with precision and with minimal blood loss [41]. Safety for an electrical diathermy cutting procedure refers to avoiding excessive contact forces while cutting a tissue or an organ. Electric diathermy cutting is particularly attractive to MIS and robotic surgery as the diathermy tips can be customized and is one of the common tools used [42]. Ex-vivo use of diathermy is also well established both for evaluation of the tool and for the training of surgeons. Using diathermy as the tool for testing the STIFF-FLOP soft robot seemed ideal due to real life similarities and applications. Challenges included customizing a widely used tool for a flexible robot arm whilst preserving maneuverability, functionality, and accuracy which has not been done before using a soft continuum robot.

The use of electrical diathermy in robotic surgery is well studied and the aim of this section is to investigate controlling a standard diathermy tip on standard tissue used for ex-vivo training. The same trials are carried out on chicken and pig tissue, to test the robustness, cutting precision and repeatability of this soft robotic instrument on animal tissues with different stiffness properties. Animal muscular tissue samples were chosen over an in-vitro rigid substrate to emphasize testing of the specific application proposed, maintaining a balance between controlled testing conditions, in terms of surface and stiffness consistency, and a realistic substrate that closely mimics tissue density of organs like the prostate, uterus or the wall of the pharynx. The tissue used corresponds to the pectoral muscle of chicken and psoas major of a pig. Further, chicken and pig muscle tissue are a commonly used substrate for training surgeons in diathermy.

The average stiffness measured during the initial contact phase for the chicken pectoral muscle is $1.836 \mathrm{~N} / \mathrm{cm}$ and that for pig psoas major is $1.434 \mathrm{~N} / \mathrm{cm}$. In order to assess the capability of the system to adapt to different target cutting trajectories, tests are performed both in straight and curved trajectories with curvature radii decreasing from $2.5 \mathrm{~cm}$ down to $1 \mathrm{~cm}$. Depending on the radius of curvature, the total length of the desired cutting path varies between $21 \mathrm{~mm}$ and $32 \mathrm{~mm}$. Every test combination, i.e. each tissue type and cut curvature, is carried out three times for statistical significance. It is pointed out that there would not be any mechanical or electrical interferences between the cutting tool and manipulator components, since the wiring of the cutting tool (currently only actuation, no sensing signals are received directly from the knife) is embedded within the soft modules, routed together

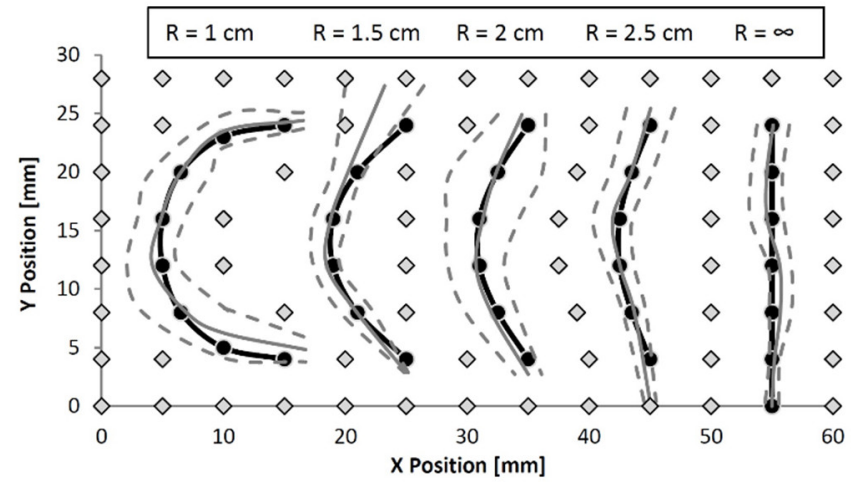

$\diamond$ Base Grid —-Target Path Mean Followed Path - - - Std. Dev. Error

Fig. 5. Template for target cutting trajectories and base grid with overlaid experimental results for mean followed path \pm one standard deviation error.

with the actuation/sensing channels of the modules with proper insulation.

The goal position of phase 1 of all experiments is defined for the insertion axis to be aligned with the desired contact position and approximately normal, i.e. angle deviations below \pm 5 degrees, to the target tissue surface, which is in a roughly horizontal position. More complex configurations and bigger misalignments would be possible thanks to the capability of the rigid-link arm to maintain its positioning accuracy regardless of the trajectory and of the soft modules to compensate the effect of gravity and to maintain their configuration using their granular jamming mechanism. However, in a more realistic scenario it is likely that the insertion port will not be aligned with the target surface and the soft robot will need to assume a complex configuration to conform to the tortuous internal shape of the organism to reach the target location.

Such conditions would require more complex sensing schemes, e.g. inter-module and skin force/torque sensors to discern interaction forces with the trocar port or en-route organs, and a more general hybrid force-motion controller, expanding the frameworks proposed in [43], [44] to multisegment soft manipulators with variable stiffness. Although such improvements would emphasize the potential superiority of soft continuum manipulators in safely following tortuous paths when navigating inside the human body, the focus of this paper and its experiments is to demonstrate the capability of the proposed system to follow 2D trajectories with its end-effector while controlling contact force matching the accuracy levels that teleoperated rigid-link surgical robots are capable of [45].

The desired trajectories are marked onto the tissue by applying ink dots, without piercing or indenting the tissue itself to avoid influencing the cut. Dots are spaced in a $4 \mathrm{~mm} \times 5 \mathrm{~mm}$ grid, using black ink for the cut path itself to guide the operator and white ink for intermediate dots for manual ground truth measurements using a $0.01 \mathrm{~mm}$ resolution digital caliper.

These measurements are performed both prior to and after each test, so as to account for tissue deformations caused by the cut when assessing its accuracy. The template grid and cutting paths used is shown in Fig. 5, together with samples on chicken tissue of (b) curved and (c) straight trajectories before and after carrying out the cutting test. As expected, the tissue in contact or close vicinity to the cut trajectory was deformed due to the 

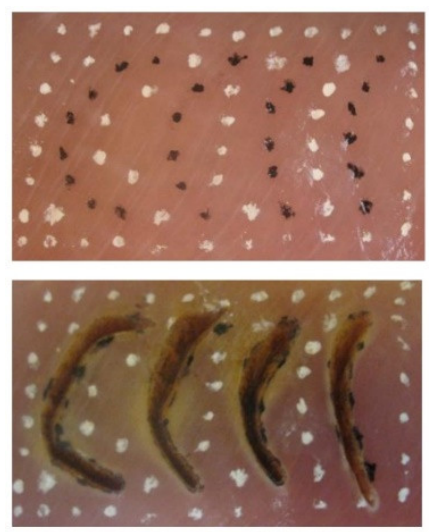

(b)

Fig. 6. Cutting paths on chicken tissue samples with (b) curved and (c) straight cut tests before (top) and after (bottom) the execution of the task.

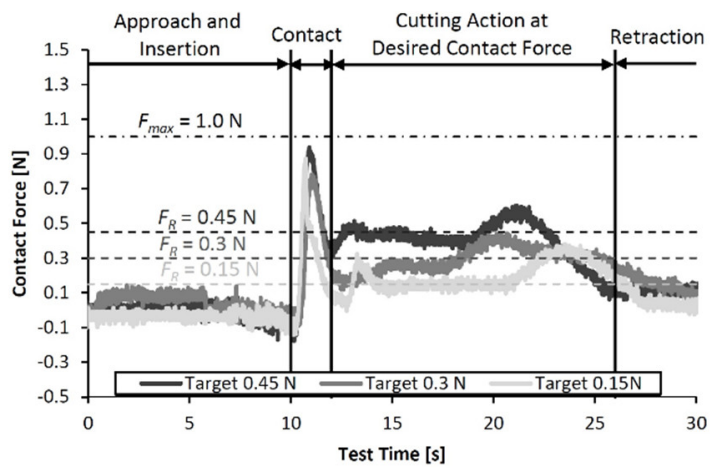

Fig. 7. Examples of the evolution of contact force versus time throughout an experiment with the three different target force values used in testing.

shrinking caused by the cutting and heating. Nevertheless, most of the points forming the "control mesh", painted in white in between the target cut trajectories, remained outside those deformed tissue regions. This can be qualitatively seen by comparing the bottom and top images (same tissue before and after the tests) in Fig. 6. Only those "control" points that were clearly unaffected by this deformation were used to measure the $\mathrm{XY}$ location of the edges of each cut.

\section{EXPERIMENTAL RESULTS}

Three main quantifiable aspects of the system's operation were measured during and after the experiments and analyzed to assess the suitability and efficiency of the designed solution: 1) the measured contact force relative to the desired safety range, 2) the deviations of the actual cutting path from the predefined target trajectory, and 3) the consistency of cut depth, width and speed.

\section{A. Contact Force Regulation}

The target contact force was changed to three different levels $(0.15 \mathrm{~N}, 0.3 \mathrm{~N}$ and $0.45 \mathrm{~N})$ for each of the combinations of tissue and cut curvature tested, so as to assess the capability of the contact force controller to maintain the desired safe values for a range of small forces. These values were chosen according to the safe contact force recommended for catheter insertion [46], [47], ranging from $0.1 \mathrm{~N}$ to $0.8 \mathrm{~N}$, and characterized for the operation of miniaturized cutting [48] and electrocautery [49] tools operating on muscular tissue, between $0.1 \mathrm{~N}$ and $0.4 \mathrm{~N}$.

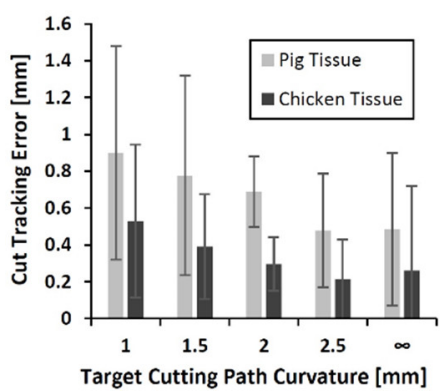

(a)

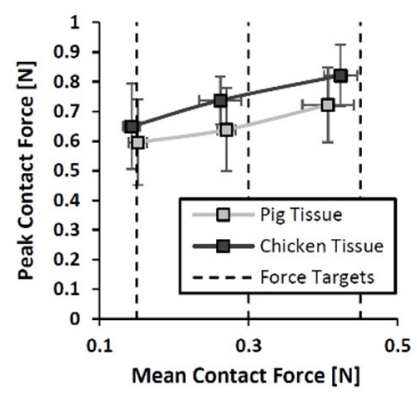

(b)
Fig. 8. Comparison of (a) the mean and peak contact forces experienced among all tests with different tissues, curvature radii and target forces and (b) the mean error between the actual and target cut path, with one standard deviation error bars, for all curvature radii and the two tissue types.

The evolution of the registered contact force through time during a test follows the same pattern regardless of the type of tissue or trajectory shape, as shown by the sample curves shown in Fig. 7. The force value remains close to zero during the first phase and most of the second phase of the task, until the electrosurgical knife touches the tissue. A steep rise ensues, which is rapidly counteracted by the force regulation to achieve the selected contact force. The overshoot phase is due to the gains chosen for the PID controller used to regulate the normal contact force, as described in sub-section III.A. It lasts under two seconds, as per the target settling time mentioned in III.A. This overshoot could be avoided by tuning the parameters of the PID controller to be less aggressive but at the expense of a slower response time which is undesirable. A trade-off was conducted to select suitable values of the controller gains without compromising much on the speed of tissue cutting.

The contact force is successfully maintained close to the desired values, marked by horizontal dashed lines in Fig. 7 and vertical dashed lines in Fig. 8(a), as the soft manipulator is actuated and the electrosurgical knife is activated to produce the cut. A minor rise of the contact force usually appears towards the end of the cut, when initiating the retraction of the tool, but it always remains well below the peak reached during the initial contact phase and within the specified $1 \mathrm{~N}$ safety limit.

On average, the contact force measured during the cutting action was consistently close to the target, but generally slightly lower as shown in Fig. 8(a). This contributes to safer operation, together with the ubiquitous satisfaction of the $1 \mathrm{~N}$ maximum force threshold, as depicted in the same graph.

The peak forces do experience a steady growth as the target force is increased, raising the possibility of eventually surpassing the $1 \mathrm{~N}$ threshold when further increasing the target force value. No statistically significant difference is observed between the two types of tissue tested, although peak forces where higher on average with chicken tissue.

\section{B. Cut Tracking Accuracy}

When it comes to the accuracy with which the tele-operated system was able to follow the desired cut trajectory, no significant errors were experienced. As seen in Fig.5, which overlays the mean executed cuts with one standard deviation intervals on the desired paths, the experimental results follow the targeted paths accurately. Therefore, the teleoperation 
TABLE I

SUMMARY OF DIATHERMY TISSUE CUTTING EXPERIMENTS

\begin{tabular}{l|c|c|c|c|c|c}
\hline \hline \multicolumn{1}{c|}{ Magnitudes } & \multicolumn{3}{|c|}{ Pig Tissue } & \multicolumn{3}{c}{ Chicken Tissue } \\
\hline Force [N] & 0.15 & 0.30 & 0.45 & 0.15 & 0.30 & 0.45 \\
\hline Force Error [\%] & 0.97 & -9.85 & -9.61 & -4.63 & -12.66 & -5.89 \\
$( \pm$ std. dev.) & $( \pm 8.1)$ & $( \pm 4.1)$ & $( \pm 7.7)$ & $( \pm 7.8)$ & $( \pm 9.5)$ & $( \pm 4.9)$ \\
\hline Cut Depth [mm] & 6.11 & 7.14 & 8.95 & 5.28 & 6.46 & 8.36 \\
( \pm std. dev.) & $( \pm 0.8)$ & $( \pm 0.9)$ & $( \pm 0.9)$ & $( \pm 1.9)$ & $( \pm 2.4)$ & $( \pm 2.2)$ \\
\hline Cut Width [mm] & 1.64 & 1.22 & 1.20 & 1.37 & 1.87 & 2.31 \\
$( \pm$ std. dev.) & $( \pm 0.7)$ & $( \pm 0.7)$ & $( \pm 0.3)$ & $( \pm 0.7)$ & $( \pm 0.5)$ & $( \pm 0.6)$ \\
\hline Cut Time [s] & 17.9 & 14.6 & 24.3 & 17.2 & 20.5 & 18.3 \\
$( \pm$ std. dev.) & $( \pm 4.1)$ & $( \pm 1.9)$ & $( \pm 3.7)$ & $( \pm 1.7)$ & $( \pm 1.5)$ & $( \pm 3.4)$ \\
\hline \hline
\end{tabular}

delays mentioned in sub-section IV.A, were not significant enough to result in a loss of accuracy once the user is familiar with the system. Overall, as depicted in Fig. 8(b), the mean cutting errors are below $1 \mathrm{~mm}$. These values are not expected to be significantly lower than those achieved by a human in hand-held ex-vivo diathermy cutting, but they also leave little margin for improvement under said testing conditions. They undergo a constant decrease as the curvature of the targeted path is reduced, achieving minimum errors for the straight trajectories. However, these curvature-induced differences are not significant in comparison with the experimental standard deviations plotted in the same graph. Similarly, the errors on tests with chicken tissue are lower on average than those on pig tissue, but the differences between both are not significant.

The "mean path followed" overlaid in Fig. 5 and used to calculate the cut tracking errors plotted in Fig. 8(b) was estimated as the centreline equidistant from those measured cut edges. The minimization of tissue deformation effect over cut accuracy assessment was double-checked quantitatively by measuring the relative distances between the "control" points used after the test and comparing them to their values before the tests. This ensured that the variation of these relative distances was below $5 \%$ and normalizing the cut edge measurements according to those variations from the pre-to-post testing.

The path tracking errors are in the same order of magnitude as those achievable using a traditional rigid-link robot [45], validating the soft robot proposed as a potential replacement for rigid-link manipulators in the application considered.

\section{Cut Depth, Width and Speed}

The measured width of the cuts performed demonstrates high consistency throughout all tets, staying in the $1.2-2.3 \mathrm{~mm}$ range on average and without any significant influence from the tissue type or contact force applied, as summarized in Table I. The same table shows the increase of the measured cut depth as the contact force applied grows both for the pig and chicken tissue, going from 5-6 mm for a $0.15 \mathrm{~N}$ force up to $8-9 \mathrm{~mm}$ for a 0.45 $\mathrm{N}$ force. The depth is slightly smaller and increases faster with force for the chicken tissue tests, due to its higher stiffness.

Depth variability during these tests is also significantly higher, with standard deviations over twice as big as those during experiments with pig tissue, potentially due to higher tissue heterogeneity and surface unevenness. The average execution time for the full task ranged from 30 to 55 seconds, with an average of $\sim 36$ seconds. The time spent in the cutting operation itself, i.e. phase 3 , accounted for roughly half of that time, with an average of $\sim 18$ seconds to complete the cut. This amounts to an effective cutting speed of $1.39 \mathrm{~mm} / \mathrm{s}$ on average.

\section{CONCLUSION}

The outcomes of safe real-time testing and validation of a new robotic surgical manipulator, which exploits the complementary advantages of a conventional rigid-link articulated arm and a novel soft-pneumatic continuum manipulator is presented in this paper. Ex-vivo validation of the integrated system was conducted successfully by demonstrating precise tissue cutting through electrical diathermy. This research advances the state-of-the-art of robot assisted MIS procedures requiring tissue resection or ablation in regions otherwise difficult to access.

The results presented go beyond previously published research, in that they simplify the tele-operated control of the cutting tool by de-coupling safe contact force regulation, achieved through the rigid-link arm, and two-dimensional cut path tracking, achieved through the soft manipulator. They also contribute an unprecedented quantitative analysis of robotassisted diathermy cutting accuracy with a system of this type.

The effectiveness of the robotic surgical manipulator proposed was validated using laboratory experiments on exvivo animal soft tissues, and its efficiency was quantitatively evaluated in terms of its ability to control the contact force applied and to precisely track the desired cutting trajectory. The system is able to track desired small forces, both avoiding contact loss and excessive forces. Errors when following the path were small and robust to different cut path curvatures and two different types of tissue with different textures.

Future research will improve on the usability of the teleoperation system, making it more accessible to users who are not familiar with the master input device. Haptic and more realistic visual feedback will be included in future ex-vivo testing using other tissue types, as well as more realistic scenarios in terms of insertion angles and soft robot configuration. For this purpose, as discussed in Section IV.C, existing hybrid motion-force control approaches will be extended and generalized for multi-segment soft continuum manipulators with variable stiffness. Furthermore, additional clinical requirements will be considered to perform in-vivo testing and clinical trials, where the proposed system's advantages relative to open surgery and MIS with traditional rigid tools can be most highlighted. In future research, real-life comparison of performance between the STIFF-FLOP soft robot and surgeon will be considered for the benchmarking exercise using data from in-vivo experiments. It is envisaged that the modularity in design will enable this new generation of soft-pneumatic continuum manipulator to be used in conjunction with other well-established rigid surgical robots, such as the da Vinci robot.

\section{ACKNOWLEDGMENT}

The authors wish to acknowledge Dr. Duale Mahdi, for his contributions to the experimental setup and design, and to the members of the EU FP7 STIFF-FLOP consortium, in particular King's College London (UK) for providing their torque sensor, Przemysłowy Instytut Automatyki i Pomiarów (Poland) for providing the design of the soft manipulator modules, and Shadow Robot Company (UK) for providing the RoNeX board. 


\section{REFERENCES}

[1] G. Dogangil et al., "A Review of Medical Robotics for Minimally Invasive Soft Tissue Surgery," Journal of Engineering in Medicine, vol. 224, no. 5, pp. 653-679, May, 2010.

[2] J. Burgner-Kahrs et al., "Continuum Robots for Medical Applications: A Survey," IEEE Trans. Biomedical Engineering, vol. 31, no. 6, pp. 12611280, Dec., 2015.

[3] H. Choi et al., "Surgical Robot for Single-Incision Laparoscopic Surgery," IEEE Trans. Biomedical Engineering, vol. 61, no. 9, pp. 24582466, Sept., 2014.

[4] K. Kwok et al., "Dimensionality Reduction in Controlling Articulated Snake Robot for Endoscopy Under Dynamic Active Constraints," IEEE Trans. Robotics, vol. 29, no. 1, pp. 15-31, Feb., 2013.

[5] F. Renda et al., "Dynamic Model of a Multibend Soft Robot Arm Driven by Cables," IEEE Trans. Robotics, vol. 30, no. 5, pp. 1109-1122, Oct., 2014.

[6] S. Russo et al., "A Novel Robotic Platform for Laser-Assisted Transurethral Surgery of the Prostate," IEEE Trans. Biomedical Engineering, vol. 62, no. 2, pp. 489-500, Feb., 2015.

[7] C. D. Onal and D. Rus, "A Modular Approach to Soft Robots," in Proc. $4^{\text {th }}$ IEEE RAS/EMBS International Conference on Biomedical Robotics and Biomechatronics, Rome, Italy, June, 2012, pp. 1038-1045.

[8] P. Polygerinos et al., "Modelling of Soft Fiber-Reinforced Bending Actuators," IEEE Trans. Robotics, vol. 31, no. 3, pp. 778-789, June, 2015.

[9] H. Mochiyama and T. Suzuki, "Dynamics Modelling of a HyperFlexible Manipulator," in Proc. $41^{\text {st }}$ IEEE SICE Annual Conference, Osaka, Japan, Aug., 2002, pp. 1505-1510.

[10] K. Suzumori et al., "Development of Flexible Microactuator and its Applications to Robotic Mechanisms," in Proc. IEEE ICRA, Sacramento, USA, Apr., 1991, pp. 1622-1627.

[11] B. A. Jones and I. D. Walker, "Kinematics for Multisection Continuum Robots," IEEE Trans. Robotics, vol. 22, no. 1, pp. 43-55, Feb., 2006.

[12] B. A. Jones and I. D. Walker, "Practical Kinematics for Real-Time Implementation of Continuum Robots," IEEE Trans. Robotics, vol. 22, no. 6, pp. 1087-1099, Dec., 2006.

[13] Y. Bailly et al., "Modelling and Control of a Continuum Style Microrobot for Endovascular Surgery," IEEE Trans. Robotics, vol. 27, no. 5, pp. 1024-1030, Oct., 2011.

[14] G. S. Chirikjian, "Hyper-Redundant Manipulator Dynamics: A Continuum Approximation," Advanced Robotics, vol. 9, no. 3, pp. 217 243, June, 1994.

[15] W. Khalil et al., "Dynamic Modeling and Simulation of a 3-D Serial EelLike Robot," IEEE Trans. Systems, Man and Cybernetics, vol. 37, no. 6, pp. 1259-1268, Nov., 2007.

[16] I. S. Godage et al., "Pneumatic Muscle Actuated Continuum Arms: Modelling and Experimental Assessment," in Proc. IEEE ICRA, Minneapolis, USA, May, 2012, pp. 4980-4985.

[17] C. Pacchierotti et al., "Cutaneous Feedback of Fingertip Deformation and Vibration for Palpation in Robotic Surgery," IEEE Trans. Biomedical Engineering, vol. 63, no. 2, pp. 278-287, Feb., 2016.

[18] O. Lambrecy et al., "A Method to Study Precision Grip Control in Viscoelastic Force Fields Using a Robotic Gripper," IEEE Trans. Biomedical Engineering, vol. 62, no. 1, pp. 39-48, Jan., 2015.

[19] T. K. Adebar et al., "3-D Ultrasound-Guided Robotic Needle Steering in Biological Tissue," IEEE Trans. Biomedical Engineering, vol. 61, no. 12, pp. 2899-2910, Dec., 2014.

[20] G. Li et al., "Robotic System for MRI-Guided Stereotactic Neurosurgery," IEEE Trans. Biomedical Engineering, vol. 62, no. 4, pp. 1077-1088, Apr., 2015.

[21] S. Tully and H. Choset, "A Filtering Approach for Image-Guided Surgery with a Highly Articulated Surgical Snake Robot," IEEE Trans. Biomedical Engineering, vol. 63, no. 2, pp. 392-402, Feb., 2016.

[22] C. Staub et al., "Implementation and Evaluation of a Gesture-Based Input Method in Robotic Surgery," in Proc. IEEE International Workshop on HAVE, Quinhuangdao, China, Oct., 2011, pp. 1-7.

[23] I. Nisky et al., "Uncontrolled Manifold Analysis of Arm Joint Angle Variability During Robotic Teleoperation and Freehand Movement of Surgeons and Novices," IEEE Trans. Biomedical Engineering, vol. 61, no. 12, pp. 2869-2881, Dec., 2014.

[24] S. A. Bowyer and F. Rodriguez y Baena, "Dissipative Control for Physical Human-Robot Interaction," IEEE Trans. Robotics, vol. 31, no. 6, pp. 1281-1293, Dec., 2015.
[25] S. B. Kesner and R. D. Howe, "Robotic Catheter Cardiac Ablation Combining Ultrasound Guidance and Force Control," International Journal of Robotics Research, vol. 33, no. 4, pp. 631-644, Apr., 2014.

[26] T. Deng et al., "Development of a New Cable-Driven Soft Robot for Cardiac Ablation," in Proc. IEEE ROBIO, Shenzhen, China, Dec., 2013, pp. 728-733.

[27] T. Ranzani et al., "A soft modular manipulator for minimally invasive surgery: Design and characterization of a single module," IEEE Transactions on Robotics, vol. 32, no. 1, pp. 187-200, Feb., 2016.

[28] M. Cianchetti et al., "Soft Robotics Technologies to Address Shortcomings in Today's Minimally Invasive Surgery: The STIFF-FLOP Approach," Soft Robotics, vol. 1, no. 2, pp. 122-131, June, 2014.

[29] G. Gerboni et al., "Modular Soft Mechatronic Manipulator for Minimally Invasive Surgery (MIS): Overall Architecture and Development of a Fully Integrated Soft Module," Meccanica, vol. 50, no. 11, pp. 2865-2878, Nov., 2015.

[30] L. Margheri et al., "Soft Robotic Arm Inspired by the Octopus: I. From Biological Functions to Artificial Requirements," Bioinspiration and Biomimetics, vol. 7, no. 2, pp. 1-12, May, 2012.

[31] M. S. Malekzadeh et al., "Learning by Imitation with the STIFF-FLOP Surgical Robot: Biomimetic Approach Inspired by Octopus Movements," Robotics and Biomimetics, vol. 1, no. 13, pp. 1-15, Oct., 2014.

[32] G. Levy et al., "Arm Coordination in Octopus Crawling Involves Unique Motor Control Strategies," Current Biology, vol. 25, no. 9, pp. 1195-1200, May, 2015.

[33] M. Cianchetti et al., "STIFF-FLOP Surgical Manipulator: Mechanical Design and Experimental Characterization of the Single Module," in Proc. IEEE/RSJ IROS, Tokyo, Japan, Nov., 2013, pp. 3576-3581.

[34] J. Fras et al., "New STIFF-FLOP Module Construction Idea for Improved Actuation and Sensing," in Proc. IEEE ICRA, Seattle, USA, May, 2015, pp. 2901-2906.

[35] N. G. Cheng et al., "Design and Analysis of a Robust, Low-Cost, Highly Articulated Manipulator Enabled by Jamming of Granular Media," in Proc. IEEE ICRA, Minneapolis, USA, May, 2012, pp. 4328-4333.

[36] A. Jiang et al., "Design of a Variable Stiffness Flexible Manipulator with Composite Granular Jamming and Membrane Coupling," in Proc. IEEE/RSJ IROS, Vilamoura, Portugal, Oct., 2012, pp. 2922-2927.

[37] Y. Noh et al., "A Three-axial Body Force Sensor Designed for Flexible Surgical Robotic Devices," in Proc. IEEE ICRA, Hong Kong, China, June, 2014, pp. 6388-6393.

[38] S. M. Mustaza et al., "Tunable Stiffness Design of Soft Continuum Manipulator," in Intelligent Robotics and Applications - Proc. $8^{\text {th }}$ ICIRA, Portsmouth, UK, Aug., 2015, pp. 152-163.

[39] "Ronex board by Shadow Robot." [Online]. Available: http://www.shadowrobot.com/products/ronex/ (Date last accessed: 04/04/2016)

[40] S. Garrido-Jurado et al., "Automatic Generation and Detection of Highly Reliable Fiducial Markers under Occlusion," Pattern Recognition, vol. 47, no. 6, pp. 2280-2292, June, 2014.

[41] "Principles of Electrosurgery." Megadyne ${ }^{\mathrm{TM}}$, 2013, available from http://www.megadyne.com/pdf/electrosurgery-principles.pdf

[42] N. Francis et al. (Eds.), "Training in Minimal Access Surgery," Springer, 2015, ISBN 978-1-4471-6494-4.

[43] O. Khatib, "A unified approach for motion and force control of robot manipulators: The operational space formulation," IEEE Journal on Robotics and Automation, vol. 3, no. 1, pp. 43-53, 1987.

[44] A. Bajo, and S. Nabil. "Hybrid motion/force control of multi-backbone continuum robots," International Journal of Robotics Research, vol. 35, no. 4, pp 422-434, 2016.

[45] L. Wang et al., "Force-Controlled Exploration for Updating Virtual Fixture Geometry in Model-Mediated Telemanipulation," Journal of Mechanisms and Robotics, vol. 9, no. 2, 2017.

[46] C. Tercero et al., "Autonomous catheter insertion system using magnetic motion capture sensor for endovascular surgery," Int. Journal of Medical Robotics and Computer Assisted Surgery, vol. 3, no. 3, pp. 52-58, 2007.

[47] U. Seibold et al., "Prototypic Force Feedback Instrument for Minimally Invasive Robotic Surgery", Medical Robotics, V. Bozovic (Ed.), InTech, DOI: 10.5772/5262, available from: https://www.intechopen.com/books/

[48] P. Valdastri et al., "Miniaturized cutting tool with triaxial force sensing capabilities for minimally invasive surgery," ASME Trans. Journal of Medical Devices, vol. 1, no. 3, pp. 206-2011, September 2007.

[49] G. J. Ball et al, "Snap-on Robotic Wrist Module for Enhanced Dexterity in Endoscopic Surgery," in Proc. IEEE ICRA, Stockholm, Sweden, May, 2016, pp. 4398-4405. 